\title{
Compressive Sensing Based Grant-Free Random Access for Massive MTC
}

\author{
Yikun $\mathrm{Mei}^{*}$, Zhen Gao*, De $\mathrm{Mi}^{\dagger}$, Pei Xiao ${ }^{\dagger}$, Mohamed-Slim Alouini ${ }^{\ddagger}$ \\ ${ }^{*}$ School of Information and Electronics, Beijing Institute of Technology, Beijing 100081, China \\ $\dagger 5$ G Innovation Centre (5GIC), Institute for Communications Systems, University of Surrey, Guildford GU2 7XH, U.K. \\ $\ddagger$ Division of Computer, Electrical and Mathematical Sciences, and Engineering, \\ King Abdullah University of Science and Technology, Thuwal 23955-6900, Saudi Arabia \\ Email: gaozhen16@bit.edu.cn
}

\begin{abstract}
Massive machine-type communications (mMTC) are expected to be one of the most primary scenarios in the next-generation wireless communications and provide massive connectivity for Internet of Things (IoT). To meet the demanding technical requirements for mMTC, random access scheme with efficient joint activity and data detection (JADD) is vital. In this paper, we propose a compressive sensing (CS)-based grant-free random access scheme for $\mathrm{mMTC}$, where JADD is formulated as a multiple measurement vectors (MMV) CS problem. By leveraging the prior knowledge of the discrete constellation symbols, we develop an orthogonal approximate message passing (OAMP)-MMV algorithm for JADD, where the structured sparsity is fully exploited for enhanced performance. Moreover, expectation maximization (EM) algorithm is employed to learn the unknown sparsity ratio of the a priori distribution and the noise variance. Simulation results show that the proposed scheme achieves superior performance over other state-of-the-art CS schemes.
\end{abstract}

Index Terms-Compressive sensing, massive machine-type communications, orthogonal approximate message passing, multiple measurement vectors.

\section{INTRODUCTION}

Massive machine-type communications (mMTC) are poised to provide ubiquitous and unprecedent connectivity for billions of Internet of Things (IoT) devices [1], [2]. To this end, new random access scheme with efficient joint activity and data detection (JADD) is desirable in order to improve reliability and simplify signaling overhead.

Compared with the current downlink-dominated wireless network, the mMTC invoked by massive potential IoT devices are uplink-dominated and have the features of sporadic traffic with low rates and short package [1], [2]. Therefore, for the mMTC scenario, the current grant-based random access schemes, such as long term evolution (LTE), suffer from the low access efficiency and high latency caused by the complicated handshake procedure [3]. As a promising alternative, grant-free random access scheme has attracted significant attention, where the devices can directly transmit data to the base station (BS) without complicated handshaking process [5]-[9].

On the other hand, due to the limited available spectrum, most grant-free access solutions have to rely on nonorthogonal radio resources. In this case, how to optimize JADD is a challenging issue. By exploiting the inherent sparsity of sporadic traffic in mMTC, the JADD can be formulated as a compressive sensing (CS) problem and solved by typical sparse signal recovery algorithms [5]-[10]. The prior work [4] has demonstrated that the CS-based multi-user detector can outperform the traditional linear minimum mean square error (LMMSE) detector when the level of user activity is low. Furthermore, to exploit the temporal partial correlation of device activity, a modified subspace pursuit (SP) algorithm and a modified orthogonal match pursuit (OMP) algorithm were proposed in [5] and [6], respectively, where the support estimated previously was regarded as prior knowledge in the next detection. In other work [7], [8], the device activity was considered to remain unchanged during one frame so that the block sparsity of random access signals in multiple slots was leveraged for improved performance. However, the prior knowledge of discrete constellation symbols was not utilized in [5]-[8], and the matrix inversion that incurs high computational complexity was inevitable. The joint-expectation maximization-approximate message passing (joint-EM-AMP) algorithm in [9] can effectively reduce the computational complexity and learn the activity parameters by EM algorithm, but the noise variance was required to be known. Similarly, the maximum a posteriori probability (MAP)-based iteration detection scheme presented in [10] also required the noise variance and user activity probability as prior knowledge.

In this paper, we design a beacon-aided grant-free massive access scheme based on the orthogonal frequency division multiplexing (OFDM) systems for mMTC. An orthogonal AMP-multiple measurement vectors (OAMP-MMV) algorith$\mathrm{m}$ is developed for JADD, where the prior knowledge of discrete constellation symbol and the structured sparsity of MMV model are fully exploited for improved performance. Moreover, the unknown sparsity ratio of a priori distribution and the noise variance can be learned by EM algorithm. Simulation results demonstrate the superiority of the proposed OAMP-MMV algorithm over state-of-the-art schemes.

Notation: The boldface lower and upper-case symbols denote column vectors and matrices, respectively. $(\cdot)^{*},(\cdot)^{\mathrm{T}}$, $(\cdot)^{\mathrm{H}},(\cdot)^{-1}, \mathrm{E}[\cdot]$ and $\operatorname{var}[\cdot]$ denote the conjugate, transpose, conjugate transpose, inversion, expectation and variance operators, respectively. $\|\mathbf{a}\|_{2}$ is the $\ell_{2}$ norm of $\mathbf{a} \cdot \operatorname{diag}(\mathbf{a})$ is a diagonal matrix with elements of $\mathbf{a}$ on its diagonal. $\mathbf{I}_{n}$ denotes 
the identity matrix of size $n \times n$, and $\mathbf{0}_{n}$ denotes the vector of size $n$ with all the elements being 0 . $\mathbf{a} \sim \mathcal{C N}(\boldsymbol{\mu}, \boldsymbol{\Gamma})$ denotes that a obeys the complex Gaussian distribution with mean vector $\boldsymbol{\mu}$ and covariance matrix $\Gamma$. $\Re \mathfrak{e}\{\cdot\}$ denotes the real part of the corresponding arguments and $\operatorname{supp}\{\cdot\}$ denotes the set of non-zero elements in a vector.

\section{System Model}

The system model of grant-free access scheme for mMTC is presented in this section. As shown in Fig. 1(a), a singleantenna BS serves $K$ single-antenna IoT devices, where $K$ can be very large but only $K_{a}\left(K_{a} \ll K\right)$ devices are active in each OFDM symbol interval. The $k$-th device transmits data symbol $x_{k, t}$ during the $t$-th OFDM symbol, where $x_{k, t}$ comes from a modulation constellation set $\Omega=\left\{a_{1}, a_{2}, \cdots, a_{L}\right\}$. If the device is silent in this period, then $x_{k, t}$ equals zero, i.e., no signal is transmitted. To distinguish the signals of the $k$ th device, $x_{k, t}$ is spread to $M$ subcarriers through a unique spreading sequence $\mathbf{s}_{k} \in \mathbb{C}^{M \times 1}$. At the BS, the received signal $\mathbf{y}_{t} \in \mathbb{C}^{M \times 1}$ can be written as

$$
\begin{aligned}
\mathbf{y}_{t} & =\sum_{k=1}^{K} \mathbf{H}_{k} \mathbf{s}_{k} \alpha_{k, t} x_{k, t}+\mathbf{w}_{t} \\
& =\mathbf{S} \mathbf{x}_{t}+\mathbf{w}_{t},
\end{aligned}
$$

where $\mathbf{H}_{k}=\operatorname{diag}\left(\left[h_{1, k}, h_{2, k}, \cdots, h_{M, k}\right]^{\mathrm{T}}\right) \in \mathbb{C}^{M \times M}, h_{m, k}$ is the $m$-th subchannel between the $k$-th device and $\mathrm{B}$ $\mathrm{S}, \alpha_{k, t}$ is the binary activity indicator that equals one when the $k$-th device is active and zero otherwise, $\mathbf{w}_{t} \sim \mathcal{C N}\left(\mathbf{0}_{M}, \sigma^{2} \mathbf{I}_{M}\right)$ is the additive white Gaussian noise, $\mathbf{S}=\left[\mathbf{H}_{1} \mathbf{s}_{1}, \mathbf{H}_{2} \mathbf{s}_{2}, \cdots, \mathbf{H}_{K} \mathbf{s}_{K}\right] \in \mathbb{C}^{M \times K}$ and $\mathbf{x}_{t}=$ $\left[\alpha_{1, t} x_{1, t}, \alpha_{2, t} x_{2, t}, \cdots, \alpha_{K, t} x_{K, t}\right]^{\mathrm{T}} \in \mathbb{C}^{K \times 1}$.

Compared to the massive number of potential IoT devices in mMTC, the number of radio resources for random access $M$ can be limited, i.e., $M \ll K$. In this case, traditional linear detectors such as least-squares and LMMSE detectors fail to solve this under-determined problem (1). However, due to the sporadic traffic, the number of active devices $K_{a}$ is much smaller than $K$, which indicates that the data vector $\mathbf{x}_{t}$ is sparse. In view of this fact, the JADD can be formulated as a single measurement vector (SMV) CS problem and can be solved by some typical sparse signal recovery algorithms. Furthermore, by stacking the received signals in multiple continuous OFDM symbols, we can formulate the JADD as an MMV CS problem to leverage the structured sparsity for enhanced performance, which will be described in the next section.

\section{PRoposed SCHEME}

\section{A. Overall Framework}

The frame structure of the proposed beacon-aided grantfree random access protocol can be shown in Fig. 1(b). Specifically, the BS periodically broadcasts a beacon for facilitating the synchronization and channel estimation at the devices. Define the estimated channel $\hat{\mathbf{H}}_{k}=\operatorname{diag}\left(\left[\hat{h}_{1, k}, \hat{h}_{2, k}, \cdots, \hat{h}_{M, k}\right]^{\mathrm{T}}\right) \in \mathbb{C}^{M \times M}$, we assume

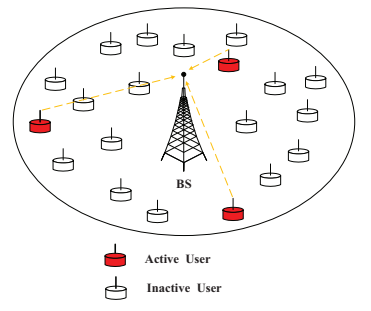

(a)

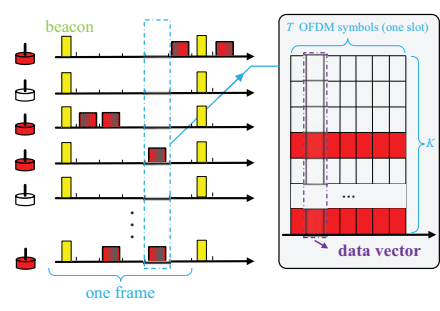

(b)
Fig. 1. (a) Active devices in mMTC are sparse. (b) For the proposed frame structure, the beacon is periodically broadcast by the BS, and the activity of devices remains unchanged in $T$ OFDM symbols.

that $\mathbf{H}_{k} \hat{\mathbf{H}}_{k}^{-1}=\mathbf{I}_{M}$ for simplicity. By taking the preequalization at the devices, we have the equivalent sensing matrix

$$
\begin{aligned}
\tilde{\mathbf{S}} & =\left[\mathbf{H}_{1} \hat{\mathbf{H}}_{1}^{-1} \mathbf{s}_{1}, \mathbf{H}_{2} \hat{\mathbf{H}}_{2}^{-1} \mathbf{s}_{2}, \cdots, \mathbf{H}_{K} \hat{\mathbf{H}}_{K}^{-1} \mathbf{s}_{K}\right] \\
& =\left[\mathbf{s}_{1}, \mathbf{s}_{2}, \cdots, \mathbf{s}_{K}\right] .
\end{aligned}
$$

At the BS, by stacking the received data signals in $T$ continuous OFDM symbols, we have

$$
\mathbf{Y}=\tilde{\mathbf{S}} \mathbf{X}+\mathbf{W}
$$

where $\mathbf{Y}=\left[\mathbf{y}_{1}, \mathbf{y}_{2}, \cdots, \mathbf{y}_{T}\right] \in \mathbb{C}^{M \times T}, \mathbf{X}=\left[\mathbf{x}_{1}, \mathbf{x}_{2}\right.$, $\left.\cdots, \mathbf{x}_{T}\right] \in \mathbb{C}^{K \times T}$ and $\mathbf{W}=\left[\mathbf{w}_{1}, \mathbf{w}_{2}, \cdots, \mathbf{w}_{T}\right]$. It is assumed that the activity of devices remains unchanged in $T$ continuous OFDM symbols, i.e.,

$$
\operatorname{supp}\left\{\mathbf{x}_{1}\right\}=\operatorname{supp}\left\{\mathbf{x}_{2}\right\}=\cdots=\operatorname{supp}\left\{\mathbf{x}_{T}\right\} \text {. }
$$

Given $\tilde{\mathbf{S}}$ and $\mathbf{Y}$, the JADD is to estimate sparse $\mathbf{X}$ and identify the active devices, which is an MMV CS problem. By leveraging the sparsity structure in (4) as prior knowledge, we can obtain the more accurate identification of active devices and improved data detection.

Besides, the selection of spreading matrix impacts greatly on the sparse signal recovery performance. Usually, both Gaussian random matrix and partial unitary matrix ${ }^{1}$ can be selected as sensing matrix $\tilde{\mathbf{S}}$ [11]. Particulary, we adopt the partial discrete Fourier transform (DFT) matrix as the sensing matrix due to the following two reasons: (a) matrix inversion can be avoided; (b) Unlike Gaussian random matrix with arbitrary values, DFT matrix is rhythmic and determinate, which facilitates quantization and storage.

The generation of partial DFT matrix is described as follows. Define $\mathbf{F} \in \mathbb{C}^{K \times K}$ as a DFT unitary matrix, i.e., $\mathbf{F F}^{\mathrm{H}}=\mathbf{I}_{K}, \mathbf{P} \in \mathbb{C}^{M \times K}$ as a selection matrix generated by randomly extracting $M$ rows from $\mathbf{I}_{K}$, we have $\tilde{\mathbf{S}}=\mathbf{P F}$. This definition will be applied in the sequel.

\section{B. OAMP-MMV Algorithm for JADD}

To leverage the sparsity structure in (4), we propose an OAMP-MMV algorithm developed from OAMP algorithm [11] for improved performance. The OAMP-MMV algorithm is firstly summarized in Algorithm 1. Assume the distribution

\footnotetext{
${ }^{1} \mathrm{~A}$ matrix $\mathbf{A} \in \mathbb{C}^{M \times K}(M<K)$ is termed as partial unitary matrix if it satisfies $\mathbf{A A}^{\mathrm{H}}=\mathbf{I}_{M}$.
} 


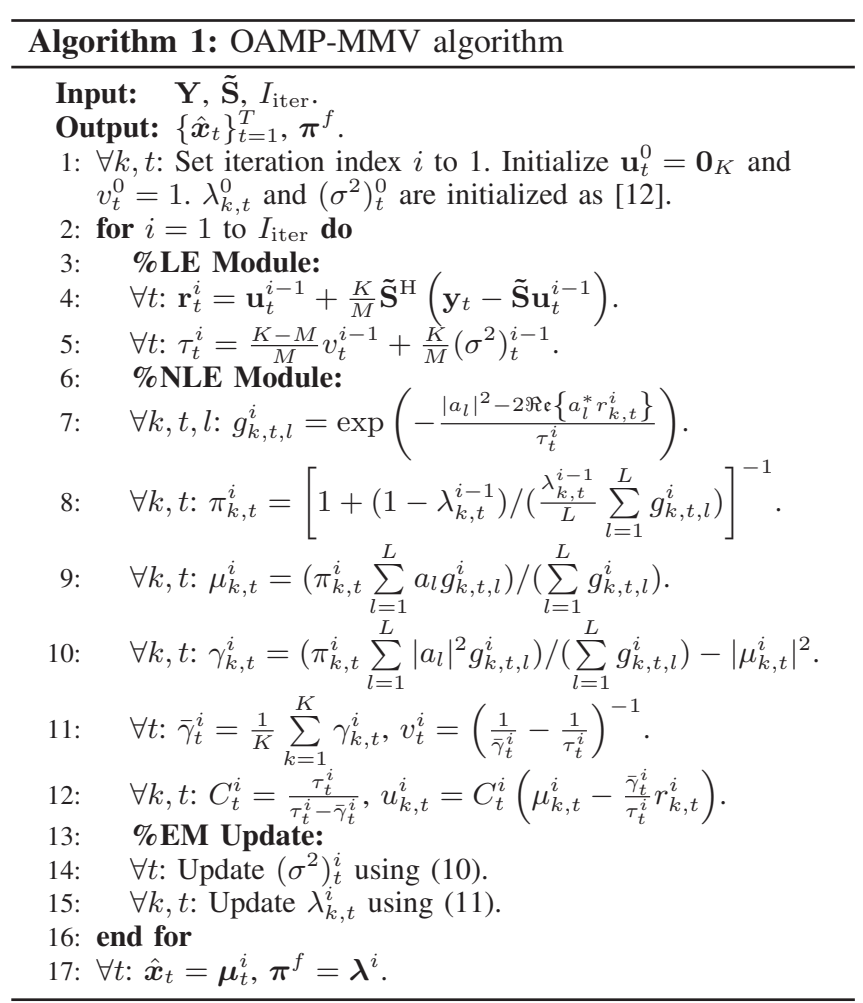

of data symbol sent by the $k$-th device during the $t$-th OFDM symbol as

$$
p\left(x_{k, t}\right)=\left(1-\lambda_{k, t}\right) \delta\left(x_{k, t}\right)+\frac{\lambda_{k, t}}{L} \sum_{l=1}^{L} \delta\left(x_{k, t}-a_{l}\right),
$$

where $\lambda_{k, t} \in[0,1]$ denotes the non-zero probability of $x_{k, t}$ and can be termed as the sparsity ratio, $\delta(\cdot)$ denotes the Dirac function.

By combining the a priori distribution in (5) with additive Gaussian noise model in [11, (16)], we can derive the approximate posterior distribution of $x_{k, t}$ as

$p\left(x_{k, t} \mid r_{k, t}\right)=\left(1-\pi_{k, t}\right) \delta\left(x_{k, t}\right)+\pi_{k, t} \frac{\sum_{l=1}^{L} g_{k, t, l} \delta\left(x_{k, t}-a_{l}\right)}{\sum_{l=1}^{L} g_{k, t, l}}$,

where $r_{k, t}, g_{k, t, l}$ and $\pi_{k, t}$ are given in lines 4,7 and 8 of Algorithm 1, respectively. Since the position of $\pi_{k, t}$ in the posterior distribution is corresponding to $\lambda_{k, t}$ in the a priori distribution, $\pi_{k, t}$ can be termed as the posterior sparsity ratio. Based on (6), the MMSE estimation can be obtained as the final output.

In Algorithm 1, lines 4-5 make up the linear estimation (LE) module of OAMP algorithm. Lines 7-12 show the nonlinear estimation (NLE) module, where the posterior mean $\mu$ and variance $\gamma$ are calculated in lines 9 and 10, respectively. The output is the posterior mean $\boldsymbol{\mu}$ in the final stage. Here we omit the detailed derivation.
Note that taking the noise variance $\sigma^{2}$ and sparsity ratio $\lambda$ as a priori knowledge is unavailable in practice. In this case, the EM algorithm [12] can be exploited to learn the unknown parameters, e.g., $\boldsymbol{\theta}=\left\{\sigma_{t}^{2}, \lambda_{k, t}, \forall k, t\right\}$, through two iterative steps

$$
\begin{aligned}
Q\left(\boldsymbol{\theta}, \boldsymbol{\theta}^{i}\right) & =\mathrm{E}\left[\ln p(\mathbf{X}, \mathbf{Y}) \mid \mathbf{Y} ; \boldsymbol{\theta}^{i}\right], \\
\boldsymbol{\theta}^{i+1} & =\arg \max _{\boldsymbol{\theta}} Q\left(\boldsymbol{\theta}, \boldsymbol{\theta}^{i}\right),
\end{aligned}
$$

where $i$ denotes the $i$-th iteration, $\mathrm{E}\left[\cdot \mid \mathbf{Y} ; \boldsymbol{\theta}^{i}\right]$ denotes the expectation conditioned on $\mathbf{Y}$ with parameters $\boldsymbol{\theta}^{i}$. The posterior distribution in (7) can be approximately replaced by (6). The joint optimization of $\boldsymbol{\theta}$ is intractable, we thus adopt the incremental EM algorithm in our scheme, where one parameter is updated at a time with other parameters fixed as constants. By taking the partial derivative of (7) with respect to each element of $\boldsymbol{\theta}$ and zeroing the derivatives, the update rules of $\boldsymbol{\theta}$ can be obtained as

$$
\begin{aligned}
\lambda_{k, t}^{i+1} & =\pi_{k, t}^{i}, \quad \forall k, t, \\
\left(\sigma^{2}\right)_{t}^{i+1} & =\frac{1}{M}\left[\left\|\mathbf{y}_{t}-\tilde{\mathbf{S}} \boldsymbol{\mu}_{t}^{i}\right\|_{2}^{2}+\frac{M}{K} \sum_{k=1}^{K} \gamma_{k, t}^{i}\right], \quad \forall t .
\end{aligned}
$$

The initialization of EM algorithm can be referred to [12].

It can be observed that $\lambda_{k, t}$ in (9) is independently updated. Since $\lambda_{k, t}$ represents the non-zero probability of $x_{k, t}$, the update rule of $\lambda_{k, t}$ can be improved by leveraging the sparsity structure for enhanced performance. According to (4), we assume that the transmitted signals from the $k$-th device in $T$ continuous OFDM symbols have the same sparsity ratio. Therefore, in the proposed OAMP-MMV algorithm, we define $\lambda_{k, t}$ as

$$
\lambda_{k, 1}^{i+1}=\lambda_{k, 2}^{i+1}=\cdots=\lambda_{k, T}^{i+1}=\frac{1}{T} \sum_{t=1}^{T} \pi_{k, t}^{i} .
$$

The joint manipulation of posterior sparsity ratio $\pi_{k, t}$ ensures that the sparsity structure of MMV problem can be learned in the EM update rules and exploited in the MMSE estimation of the next iteration.

The activity identification can be obtained according to the posterior sparsity ratio $\pi_{k, t}$ output from the proposed OAMP-MMV algorithm. In our scheme, a hard thresholdbased activity detector is developed as follows

$$
\hat{\alpha}_{k}=\left\{\begin{array}{cc}
1, \quad \pi_{k}^{f} \geq T_{h}, \\
0, \quad \text { otherwise, }
\end{array} \quad \forall k,\right.
$$

where $\hat{\alpha}_{k}=1$ indicates that the $k$-th device is active, $\pi_{k}^{f}$ is the posterior sparsity ratio in the final stage and $T_{h}$ is a threshold determined empirically.

\section{Simulation Results}

In this section, we evaluate the performance of the proposed OAMP-MMV algorithm for JADD in the grant-free massive access. In simulations, we consider $K=500$ potential IoT devices and only $K_{a}=50$ of them are active. The number of measurements $M=70$ and the active devices' data adopts the modulation of QPSK. The threshold of activity detector 


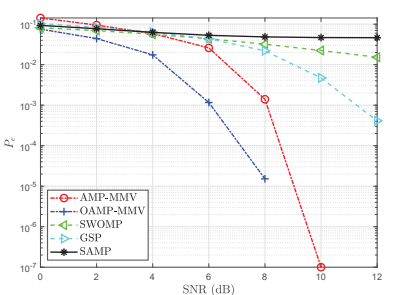

(a)

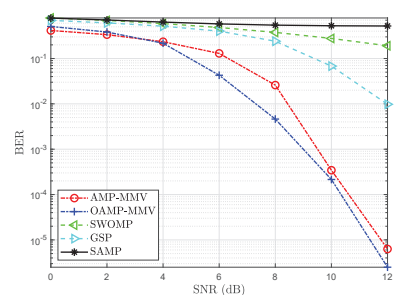

(b)
Fig. 2. Comparison of the different detection algorithms versus SNR with $T=10$ : (a) $P_{e}$; (b) BER.

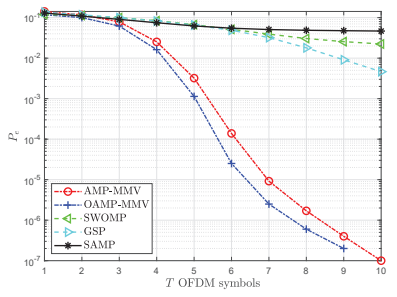

(a)

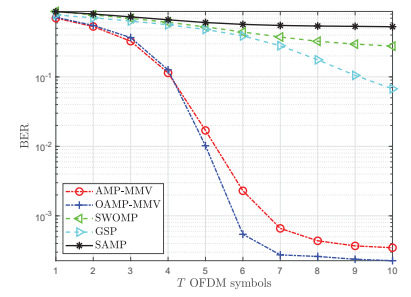

(b)
Fig. 3. Comparison of the different detection algorithms versus $T$ continuous OFDM symbols with SNR $=10 \mathrm{~dB}$ : (a) $P_{e}$; (b) BER.

$T_{h}$ is set to $1 / 3$, implying that the cost of missed detection is higher than that of false alarm. We define the error detection probability of activity detection $\left(P_{e}\right)$ and uncoded bit error rate (BER) as follows

$$
P_{e}=\frac{1}{K} \sum_{k=1}^{K}\left|\hat{\alpha}_{k}-\alpha_{k}\right|, \mathrm{BER}=1-\frac{N_{s}}{K_{a} T \log _{2} L},
$$

where $N_{s}$ denotes the number of correctly decoded bits of successfully detected devices. Moreover, the considered baseline algorithms includes AMP-MMV algorithm [13], simultaneous weighted OMP (SWOMP) algorithm [14], generalized SP (GSP) algorithm [15] and sparsity adaptive matching pursuit (SAMP) algorithm [16], where the AMP-MMV algorithm applies (11) to leverage the sparsity structure. The number of iterations of AMP-MMV and OAMP-MMV algorithms is set to 30 .

Fig. 2 verifies the superiority of the proposed OAMPMMV algorithms against different signal-noise-ratios (SNR), given the number of continuous OFDM symbols $T=10$. It can be observed that the AMP-MMV and OAMP-MMV algorithms distinctly outperform the other three algorithms, due to full exploitation of the prior knowledge of discrete constellation symbols and the sparsity structure of MMV problem. Moreover, the OAMP-MMV algorithm outperforms the AMP-MMV algorithm due to its better robustness to nonGaussian sensing matrix. By contrast, the greedy algorithms have poor performance even when SNR is relatively high.

Fig. 3 shows the performance against different $T$, when $\mathrm{SNR}=10 \mathrm{~dB}$. Note that all algorithms perform poorly when $T=2$. The rationale is that the MMV model boils down to the SMV model when $T$ is much close to 1 , resulting in loss of sparsity structure. As $T$ increases, the proposed
OAMP-MMV algorithm becomes superior to other baseline algorithms. When $T$ is larger than 9, the OAMP-MMV algorithm achieves perfect activity identification, as shown in Fig. 3(a), which demonstrates its significant gain by exploiting sparsity structure.

\section{Conclusions}

In this paper, we propose a beacon-aided uplink grantfree massive access scheme for mMTC. By formulating the JADD as an MMV CS problem, we propose an OAMP-MMV algorithm, where the prior knowledge of discrete constellation symbols and the sparsity structure is fully exploited for enhanced performance. In addition, the unknown sparsity ratio of a priori distribution and the noise variance can be learned through EM algorithm. Simulation results prove the effectiveness of the proposed scheme and demonstrated its superiority over its conventional counterparts.

\section{REFERENCES}

[1] C. Bockelmann, N. Pratas, H. Nikopour, K. Au, T. Svensson, C. Ste fanovic, P. Popovski, and A. Dekorsy, "Massive machine-type communications in 5G: Physical and MAC-layer solutions," IEEE Commun. Mag., vol. 54, no. 9, pp. 59-65, Sep. 2016.

[2] F. Ghavimi and H. H. Chen, "M2M communications in 3GPP LTE/LTEA networks: Architectures, service requirements, challenges, and applications," IEEE Commun. Surveys Tut., vol. 17, no. 2, pp. 525-549, 2nd Quart. 2015.

[3] J. Wang, Z. Zhang, and L. Hanzo, "Joint active user detection and channel estimation in massive access systems exploiting Reed-Muller sequences," IEEE J. Sel. Topics Signal Process., vol. 13, no. 3, pp. 739752, Jun. 2019.

[4] B. Shim and B. Song, "Multiuser detection via compressive sensing," IEEE Commun. Lett., vol. 16, no. 7, pp. 972-974, Jul. 2012.

[5] Y. Du et al., "Efficient multi-user detection for uplink grant-free NOMA: Prior-information aided adaptive compressive sensing perspective," IEEE J. Sel. Areas Commun., vol. 35, no. 12, pp. 2812-2828, Dec. 2017.

[6] B. Wang, L. Dai, Y. Zhang, T. Mir, and J. Li, "Dynamic compressive sensing-based multi-user detection for uplink grant-free NOMA," IEEE Commun. Lett., vol. 20, no. 11, pp. 2320-2323, Nov. 2016.

[7] B. Wang, L. Dai, T. Mir, and Z. Wang, "Joint user activity and data detection based on structured compressive sensing for NOMA," IEEE Commun. Lett., vol. 20, no. 7, pp. 1473-1476, Jul. 2016.

[8] Y. Du et al., "Block-sparsity-based multiuser detection for uplink grantfree NOMA," IEEE Trans. Wireless Commun., vol. 17, no. 12, pp. 7894 7909, Dec. 2018.

[9] C. Wei, H. Liu, Z. Zhang, J. Dang, and L. Wu, "Approximate message passing-based joint user activity and data detection for NOMA," IEEE Commun. Lett., vol. 21, no. 3, pp. 640-643, Mar. 2017.

[10] B. K. Jeong, B. Shim, and K. B. Lee, "MAP-based active user and data detection for massive machine-type communications," IEEE Trans. Veh. Technol., vol. 67, no. 9, pp. 8481-8494, Sept. 2018.

[11] J. Ma and L. Ping, "Orthogonal AMP," IEEE Access, vol. 5, pp. 2020 2033, 2017.

[12] J. P. Vila and P. Schniter, "Expectation-maximization Gaussian-mixture approximate message passing," IEEE Trans. Signal Proccess., vol. 61, no. 19 , pp. 4658-4672, Oct. 2013.

[13] X. Meng, S. Wu, L. Kuang, and J. Lu, "An expectation propagation perspective on approximate message passing," IEEE Signal Process. Lett., vol. 22, no. 8, pp. 1194-1197, Aug. 2015.

[14] J. Rodrłguez-Fernndez, N. Gonzlez-Prelcic, K. Venugopal, and R. W. Heath, "Frequency-domain compressive channel estimation for frequency-selective hybrid millimeter wave MIMO systems," IEEE Trans. Wireless Commun., vol. 17, no. 5, pp. 2946-2960, May 2018.

[15] J. M. Feng and C. H. Lee, "Generalized subspace pursuit for signal recovery from multiple-measurement vectors," in Proc. Wireless Commun. Network Conf., pp. 2874-2878, Apr. 2013.

[16] Z. Gao, L. Dai, Z. Wang, and S. Chen, "Spatially common sparsity based adaptive channel estimation and feedback for FDD massive MIMO," IEEE Trans. Signal Process., vol. 63, no. 23, pp. 6169-6183, Dec. 2015. 